
cultures

Les cahiers de l'Acedle

1 | 2004

Actes de la journée Métice

\title{
Innovation en éducation et innovation dans l'enseignement des langues : quels invariants?
}

\section{Élisabeth Brodin}

\section{(2) OpenEdition}

Journals

Édition électronique

URL : http://journals.openedition.org/rdlc/5331

DOI : $10.4000 /$ rdlc.5331

ISSN : 1958-5772

Éditeur

ACEDLE

Référence électronique

Élisabeth Brodin, «Innovation en éducation et innovation dans l'enseignement des langues : quels invariants? », Recherches en didactique des lanques et des cultures [En ligne], 1 | 2004, mis en ligne le 15 novembre 2004, consulté le 11 septembre 2019. URL : http://journals.openedition.org/rdlc/5331 DOI : 10.4000/rdlc.5331

Ce document a été généré automatiquement le 11 septembre 2019.

\section{$\theta \Theta \Theta \Theta$}

Recherches en didactique des langues et des cultures is licensed under a Creative Commons AttributionNonCommercial-NoDerivatives 4.0 International License 


\title{
Innovation en éducation et innovation dans l'enseignement des langues : quels invariants?
}

\author{
Élisabeth Brodin
}

\section{Introduction}

1 De la sphère entrepreneuriale aux hommes politiques, mais aussi des organisations aux administrations, on constate que tout le monde "innove" désormais, ou plus exactement tout le monde se doit d'innover. Au premier stade de la réflexion, on peut se demander s'il ne s'agit pas d'une réponse à un "impératif catégorique de modernité" ou si ce n'est pas l'imprécision du concept qui expliquerait en grande partie son succès.

Dans le secteur industriel, innover apparaît comme l'unique chance de survie pour les entreprises, qui développent ainsi de nouveaux produits ou systèmes de production, de nouvelles technologies et organisations.

3 L'institution éducative confronte à la même "injonction paradoxale" d'innover, à cause non seulement de la pression sociale mais aussi de l'inadéquation des dispositifs éducatifs dans certains contextes.

4 Pourtant, si la perception de l'innovation est actuellement positive dans le contexte éducatif, ce ne fut pas toujours le cas dans le passé (Cros, 2001).

5 Et même associée à l'idée de progrès, de créativité, l'innovation est génératrice de tension, porteuse de crise et souvent considérée comme un outil à l'usage des ministres qui veulent justifier leur passage éphémère ou à celui des technocrates pour montrer leur raison d'être. Portée par un ministère, l'innovation-acte politique est une réforme, qui procède, par essence, de cette "destruction créatrice" dont parlait Schumpeter dans les années 30 (cité par Alter, 2000). 


\section{Un cadre théorique multiréférentiel}

6 Traiter de l'innovation suppose un cadre théorique forcément multiréférentiel. Dès qu'on met en œuvre des dispositifs d'enseignement et d'apprentissage intégrant l'usage des technologies numériques en langues, trois champs de l'innovation au moins sont convoqués :

- celui de l'innovation technique,

- celui de l'innovation organisationnelle (les enseignements des théories des organisations sont particulièrement riches),

- celui de l'innovation en éducation et en formation.

7 Le premier permet de comprendre comment se construisent les usages des objets techniques (Flichy, 1994) en termes de schèmes sociaux et individuels, selon des processus d'instrumentation et d'instrumentalisation, en incluant les phénomènes de catachrèse chers aux enseignants de langue (Rabardel, 1995).

8 Les deux autres dimensions éclairent l'innovation de terrain (au niveau micro), de même que l'innovation d'origine institutionnelle (niveau macro) dans sa dimension politique éducative ou linguistique, même si Chappaz affirme que "la réflexion sur l'innovation en éducation et en formation a donné lieu à de nombreux travaux sans pour autant aboutir à des conclusions suffisamment satisfaisantes pour servir de base à la mise en place de politiques spécifiques" (Chappaz, 2002: 289). Mais doit-on attendre des réflexions théoriques qu'elles fournissent des modèles applicationnistes pour l'action?

\section{L'innovation en éducation et dans la formation en langues}

Toute innovation dans le secteur de la formation s'inscrit à la rencontre d'une volonté ou d'une reconnaissance institutionnelles et d'une initiative de terrain ; elle procède de l'une de ces dimensions. Le sort d'une telle innovation dépend de la façon dont est gérée la dialectique entre la logique descendante de l'institution, du pouvoir et celle de l'engagement librement consenti (Gather-Thurler \& Perrenoud, 2003).

10 Vue dans cette perspective, l'innovation en langues est une innovation éducative parmi d'autres dont elle partage les caractéristiques et les ambiguïtés, que nous allons rappeler.

11 Dans un deuxième temps, l'application au champ de l'enseignement des langues des théories de Kuhn et du paradigme du changement scientifique permet de dégager les moments où on a affaire à une période dite "normale" du progrès scientifique ou au contraire à une période de crise et d'innovation (Heyworth, 1999).

12 Pour illustrer mes propos je ferai référence à quatre exemples d'innovation, trois à l'initiative de l'institution: l'implantation du système Licence Master Doctorat dans l'enseignement supérieur ou $\mathrm{LMD}^{1}$, l'opération Campus Numériques ${ }^{2}$ ou $\mathrm{CN}$, l'introduction de l'enseignement des langues dans le primaire avec le site Primlangues ou LP (Primlangues, nd) et un vaste projet d'innovation dans l'enseignement des langues au lycée, au niveau d'une académie ${ }^{3}$ ou DLP, initié par une collectivité locale en liaison avec l'institution. Deux de ces opérations sont des réformes (LMD, LP), les deux autres n'en sont pas (CN, DLP). 


\section{Tentative de définition et clarification notionnelle} abondent dans la banque de données Nova (INPR, 2004). On tente aussi souvent de définir l'innovation en négatif, par ce qu'elle n'est pas. De manière plus constructive, je chercherai à préciser les aspects qui font consensus.

chercheurs du domaine considèrent que "l'innovation est un processus qui a pour intention une action de changement et pour moyen l'introduction d'un élément ou d'un système dans un contexte déjà structuré" (Cros, 2001 : 119).

On réserve généralement le concept de changement aux "transformations structurelles et donc relativement durables d'un système d'action, qu'il s'agisse d'une organisation complexe, d'un groupe ou d'une personne" (Gather-Thurler \& Perrenoud, 2003).

\section{Caractéristiques essentielles de l'innovation}

L'innovation, changement "conscient, intentionnel, volontaire et délibéré" (Cros, 2001) ou " élaboré et contrôlé" (Heyworth, 1999), naît forcément d'un besoin.

Ce besoin de changement est suscité, au niveau individuel, par une insatisfaction ressentie, des difficultés rencontrées au quotidien. Il faudra ensuite problématiser ces difficultés à résoudre, c'est-à-dire envisager les leviers sur lesquels on peut agir d'une manière raisonnée. La réflexion didactique est précieuse, à ce niveau.

18 Au niveau institutionnel une réforme, qui implique des changements fondamentaux dans les orientations d'une politique éducative, représente aussi une réponse, souvent engendrée dans l'urgence, à une crise, à l'insatisfaction d'une partie de la population à l'égard du système éducatif, à un décalage entre la demande d'éducation, de formation et les besoins. Il y a les motivations mises en avant pour conduire une réforme et les autres (pas forcément avouées) qui sont rarement tout à fait désintéressées.

Heyworth (1999) propose une formule de disposition au changement : $C=(a b d)>x^{4}$ qui met en évidence les paramètres déterminants pour l'engagement des acteurs et peut expliquer la "lassitude des innovateurs" (Alter, 2000).

Si l'innovation ne se décrète pas, elle ne se programme pas non plus, à la différence du projet. Certains chercheurs parlent de changement planifié, mais la planification évoque une préméditation totale qui confondrait l'innovation avec le projet. L'innovation diffère du projet avec lequel elle ne partage que la visée. C'est une ambiguïté de l'institution de demander des projets d'innovation avec une planification précise. Il est difficile voire impossible d'analyser un projet innovant en amont. L'innovation ne se reconnaît qu'après coup. L'expérience (CN, LP notamment) montre que la planification initiale doit être souple et pouvoir être revue en fonction des aléas qui adviennent.

21 Les objectifs initiaux avoués par l'innovateur pour s'inscrire dans le cadre institutionnel et obtenir des moyens financiers ne sont pas forcément ceux réellement atteints.

Aucune résistance à l'innovation n'est a priori irrationnelle ou réactionnaire. La résistance et le conflit sont des nécessités positives (Fullan, 1991). Il y a forcément des résistants qui ont leurs raisons, pas forcément avouables, mais réelles. Les résistances au changement vont de l'inertie, au refus actif en passant par le scepticisme ou le refus 
passif (Perrenoud, 1998). Elles se renforcent ou s'amenuisent au fil de l'implantation de la réforme et chaque phase du processus d'innovation réserve des surprises. Le LMD engendra d'abord des résistances à sa mise en place, de la part des étudiants, qui se sont transformées en un "désir" de LMD. C'est la perception qu'avaient les étudiants des implications du changement qui a évolué.

Quand on introduit un changement, ce qui se produit en définitive est la résultante de la confrontation entre les stratégies contradictoires des acteurs qui travaillent à la précipiter ou à l'empêcher. L'innovation fait donc l'objet d'une transaction, d'un compromis négocié, entre plusieurs intérêts, projets, logiques d'action. Il n'y aura jamais de consensus total dans une réforme d'envergure. Les acteurs concernés n'ont jamais tous le même rapport à une innovation définie, par exemple dans le cas de l'enseignement des langues dans le primaire (LP).

Ce qui est le plus intéressant, ce sont les stratégies d'innovation des acteurs, leurs résistances face aux innovations proposées par d'autres, les transactions implicites ou explicites qui s'engagent alors.

L'innovation, action finalisée, est sous-tendue par des valeurs qui déterminent l'action mise en place. L'innovation est une question axiologique, d'adhésion à des valeurs qui doivent être partagées entre les acteurs. Elle est reliée à des visions du monde, des stratégies plus larges. On innove pour introduire du meilleur en fonction des valeurs de référence... mais l'innovation peut aussi n'être qu'un moyen pour que rien ne change.

L'innovation est liée au risque et à l'incertitude et génère des craintes chez les enseignants. Au premier stade de l'innovation, il y a beaucoup d'inconnues et on ignore par définition ce qui va se passer. On prend des risques, on tâtonne. Cette prise de risques permet de se défaire des contraintes imposées par les structures de travail (Alter, 2000). Il s'agit de gérer l'incertitude. Mais dans les situations courantes d'enseignement, l'enseignant n'agit-il pas souvent dans l'urgence?

27 Les réformes top-down ou descendantes menacent le sens que les acteurs donnent à leur travail au quotidien. Cette menace est au cœur des difficultés et des résistances que rencontrent les projets de réforme.

28 Les rapports entre les acteurs oscillent entre deux logiques : une logique "instrumentale" qui en fait des agents au service du système, devant obéir aux prescriptions des autorités, l'autre plus " existentielle " qui a sa source dans le désir de garder son identité, sa tranquillité ou ses avantages (Gather-Thurler \& Perrenoud, 2003).

29 Au sein du système éducatif, cette dialectique de l'acteur et du système se décline diversement selon les niveaux de l'organisation, les enjeux et les contenus de l'innovation, les rapports qu'entretiennent les acteurs concernés.

Le sort d'une innovation dépend fortement du sens que lui donnent les acteurs, surtout quand il s'agit, pour eux, de changer leurs pratiques. Même les réformes de structures qui relèvent $a$ priori de l'institution peuvent être vidées de leur substance si les enseignants n'en comprennent pas le sens. Il est possible de respecter la lettre sans honorer l'esprit d'une réforme, par exemple dans le cas de l'enseignement des langues dans le primaire (LP).

31 Pour accroitre les chances de succès d'une réforme, il est nécessaire d'associer les acteurs à son pilotage, c'est le principe reconnu comme essentiel de toute réforme. Il faut un accompagnement pour aider à construire le lien entre institution et terrain, ce 
qui est souvent négligé par l'institution. Le manque d'accompagnement a nui à l'opération Campus Numériques $(\mathrm{CN})$, par exemple.

Il faut aussi une valorisation par un regard extérieur comme l'a montré le projet académique d'innovation dans l'enseignement des langues au lycée (DLP) (Brodin, 2002).

Or, une réforme se limite généralement à l'énoncé d'objectifs généraux sans donner d'indication précise sur les processus et les méthodes par lesquels ces processus pourront être atteints (Finkelsztein \& Ducros, 1996).

Pour conclure cette partie, on peut citer des propos récents du ministre à propos du LMD :

Cette innovation, je le sais, a pu susciter des inquiétudes... Il faut qu'ensemble nous les apaisions par l'explicitation, le dialogue et une certaine force de conviction. En la matière, chacun expérimente - les établissements comme le ministère - des voies nouvelles. Il n'est pas étonnant pour un chantier d'une telle ampleur que des ajustements méthodologiques soient nécessaires. Là encore le dialogue est et devra être la règle. (dépêche L'AEF du 22/04/2004, L'AEF, nd).

Le calendrier politique contraint à obtenir des résultats rapides (DLP, LP) qui bafouent souvent les savoirs que l'on a déjà sur l'innovation.

\section{Le temps comme variable essentielle du processus d'innovation}

En termes de processus, le changement passe par plusieurs étapes identifiées. L'innovation impulsée au niveau institutionnel connaît trois phases: dans un premier temps il s'agit d'expérimentations pilotes (LMD, CN, LP), dans un deuxième temps on laisse faire les innovateurs qui "donnent sens aux projets en les 'calant' par rapport aux réalités de terrain et en en imaginant les usages" (Alter, 2000). Dans un troisième temps l'innovation est institutionnalisée, autrement dit rationalisée ; elle devient obligatoire, "normalisée" (LP).

37 Au niveau macro de l'institution, l'innovation s'inscrit à la fois dans la continuité et dans la rupture en termes d'évolution du système éducatif. Avec le LMD il y a rupture dans l'organisation de l'offre de formation mais on enseigne les mêmes choses.

38 Au niveau de l'individu, l'innovation travaille dans la durée parce qu'elle suppose des changements de représentations. S'il est admis que l'innovation se joue à trois niveaux : au niveau des produits ou des services, à celui des technologies et des méthodes, au niveau des découvertes scientifiques et des évolutions de la pensée (Génelot,1992), c'est du dernier niveau que procède la difficulté à penser l'innovation en éducation.

39 Changer est un processus psychologique, éminemment personnel (Fullan, 1991). L'innovation dans les pratiques se fait lentement en prenant le rythme du changement personnel. Dans le projet DLP, il a fallu plusieurs années pour que les pratiques changent en profondeur.

40 Il faut donc du temps pour innover. On peut faire un parallèle avec l'apprentissage. Il faut une phase d'assimilation avec l'analyse de la nouveauté et une autre d'accommodation où le changement se concrétise.

41 Les changements qui concernent les pratiques dépendent totalement de l'adhésion des enseignants et de leur volonté d'acquérir les compétences correspondantes. On peut 
évoquer le rôle de la formation ainsi que celui de la création d'outils dans le cadre du projet académique d'innovation dans l'enseignement des langues au lycée (DLP).

Avec le LMD, les établissements choisissent le moment où ils basculent dans le nouveau régime: cette stratégie qui, certes, ralentit l'implantation de la réforme affaiblit les résistances dues à la précipitation.

\section{Vers une meilleure gestion de l'innovation : l'innovation gagne à être déléguée au praticien- didacticien "réflexif"} l'affinitaire", le "on est bien entre soi", formateurs comme apprenants, évoqués par Meirieu (2002). C'est le cas de l'opération Campus Numériques (CN) et du projet académique d'innovation dans l'enseignement des langues au lycée (DLP) :

- on crée des outils mais certains ne voient plus que l'outil créé et s'enferment sur lui ;

- l'innovation peut être vécue comme une gratification narcissique collective, voire comme un rejet de toute évaluation sous prétexte qu'on est innovateur donc meilleur que les autres et qu'on n'a de comptes à rendre à personne ;

- les innovateurs refusent la question de la transférabilité, ce qui signifierait perdre son statut marginal et donc de novateur ; 
- l'innovation est un espace où s'inventent des solutions qui seront tôt ou tard adoptées par le système central et institutionnalisées; elles auront acquis un caractère officiel qui promouvra l'innovation avec ses règles de fonctionnement. Mais l'innovation peut aussi demeurer une vitrine de luxe.

\section{Les spécificités de l'innovation en langues}

Si l'on examine l'innovation en langues à partir des théories du paradigme du changement de Kuhn (1972), il s'agit de savoir si l'enseignement et la didactique des langues se trouvent dans une "phase normale" du progrès scientifique ou s'ils sont dans une phase de crise à l'origine d' "innovations radicales", qui correspondrait à un changement de paradigme. On admettra qu'un paradigme est un ensemble de principes, d'idées, de règles acceptées par toute une communauté scientifique.

Pendant une période "normale" de progrès, la communauté scientifique travaille à développer des instruments qui permettent l'application des idées, principes et règles en vigueur et la résolution des problèmes qui se posent. En période de crise, les faits ne sont plus expliqués de manière satisfaisante et les découvertes scientifiques proposent un nouveau paradigme qui, après une période de forte résistance, sera accepté et deviendra le nouveau paradigme.

Il y a eu peu de périodes d'innovations "radicales", de ruptures, dans l'enseignement des langues :

- Chomsky a représenté une révolution en matière linguistique ;

- les approches communicatives ont, en leur temps, constitué une véritable innovation et représenté un nouveau paradigme dans l'enseignement des langues; elles ont connu leurs phases d'assimilation et d'accomodation-adaptation;

- pour ce qui est de l'approche naturelle de Krashen, il ne s'agissait en fait que d'une réactualisation d'anciennes méthodes, qui apparaissaient comme nouvelles aux yeux des enseignants (Ellis, 1997), ce qui signifie que cette nouveauté avait un caractère de nouveau relatif (à la différence de l'invention ou de la découverte).

D'après Ellis (ibid.), la recherche en acquisition des langues semble n'avoir généré que peu d'idées pédagogiques nouvelles et en tout cas pas d'innovations fondamentales, à la différence de la psychologie cognitive dans sa dimension psycholinguistique. Il cite l'enseignement des langues basé sur les tâches comme exemple de l'influence directe des théories psycholinguistiques de l'apprentissage des langues secondes.

Heyworth (1999) avance une insatisfaction actuelle par rapport au paradigme actuel et au modèle traditionnel (modèle "PPP" présenter, practise ou s'exercer, produire).

Cependant les changements qui apparaissent sont d'ordre organisationnel. Si l'on admet que les variables logistiques à prendre en compte dans l'enseignement des langues sont le temps, le lieu, les médias utilisés, le contenu et les acteurs, les changements concernent la modification de deux ou trois variables du modèle traditionnel comme, par exemple, dans l'enseignement secondaire un enseignement plus intensif, l'utilisation de ressources en libre accès, l'introduction d'autres matières enseignées dans la L2... Il semble cependant qu'il faille remettre ces expériences dans leur contexte pour en mesurer le degré d'innovation. L'innovation consiste alors à reconfigurer l'existant en fonction des ressources et des contraintes du contexte. 
L'innovation génère comme un langage nouveau créé grâce à une syntaxe nouvelle qui autorise un agencement différent d'éléments existants. Il y a re-création locale avec prise en compte du contexte.

Chaque élément des dispositifs fait déjà partie de la pratique professionnelle. Gérer un centre de langues requiert de la part de l'enseignant des compétences qu'il a déjà, même s'il doit en acquérir de nouvelles.

L'innovation en langues est ainsi avant tout une innovation de terrain, une innovation "perçue" qui relève d'une tradition dans cet enseignement (Ellis, 1997). Elle est perçue comme telle par les praticiens qui l'adoptent, sans être fondamentalement nouvelle, comme l'est une innovation "absolue" qui se définit par son caractère original. Ce qui est nouveau dans un contexte peut être courant dans un autre et peut même être contesté dans un troisième, voire abandonné.

Ces innovations de terrain peuvent faire l'objet d'une théorisation a posteriori, comme les tâches de "rétention d'information" (à résoudre par des questionnements divers pour pouvoir avancer dans les activités de résolution de problème), issues de l'approche communicative mais repensées à la lumière de la recherche en apprentissage des langues.

Selon cette optique, on peut penser que les innovations dans l'enseignement des langues qui s'appuient sur le multimédia et l'Internet fourniront à terme des modèles d'innovation, qui relèveront de plus en plus de l'enseignement néo-communicatif ou communicatif revisité, mais il semble que l'enseignement des langues avec les technologies numériques soit actuellement dans une période normale de développement, dans l'optique des théories de Kuhn (1972). On travaille à la définition des usages, dont certains font déjà consensus. Pour créer ces usages, qui diffèrent des simples utilisations en ce qu'ils s'inscrivent dans le temps long des pratiques stabilisées, il est nécessaire de connaître les éléments théoriques, d'utiliser ces propositions issues des avancées théoriques pour essayer de les faire passer dans la pédagogie des langues. Les technologies numériques contribuent à renforcer la fréquence de l'input oral fourni à l'apprenant dont, maintes fois, on a vérifié ou mesuré les effets dans des tests ultérieurs.

60 Pour Fullan (1991), c'est l'introduction de matériel pédagogique nouveau qui est ce qu'il y a de plus facile à mettre en œuvre. C'est sûrement vrai pour certains enseignants parce qu'elle mobilise leur créativité en nécessitant la création de tâches nouvelles, mais pas pour les autres. Le projet DLP (Brodin, 2002) l'a montré. Du reste, le matériel mis à disposition peut être utilisé dans un esprit autre que celui des concepteurs.

61 S'il n'y a pas d'innovation sans invention d'outils et de modèles, il n'y a pas non plus de modèle unique du changement et le modèle "applicationniste" n'est pas possible.

\section{Conclusion}

62 La professionnalisation des enseignants déjà évoquée plus haut passe par une évaluation du niveau d'expertise, qui elle-même passe par une connaissance des savoirs issus de la recherche.

La formation de praticiens réflexifs suppose des ponts constants entre théories et situations et entre savoirs savants et savoirs professionnels. 
64 disponibles sur les processus d'apprentissage, les limites des didactiques et les incertitudes de toute situation pédagogique et être conscient que

proposer de questionner la dialectique entre l'évolution des compétences des personnes, l'inscription dans des actions de terrain de ces nouvelles compétences sous forme d'actions dites innovantes et la capacité d'une organisation à accueillir, à faire fructifier, voire même en amont à favoriser l'émergence de ces innovations, c'est accepter de prendre à 'bras le corps' la complexité des systèmes humains (Chappaz, 2002: 289).

L'innovation est le moteur du développement de l'entreprise et il y existe une dynamique permanente basée sur la nouveauté, selon un processus d'innovation continu ; l'invention pour les économistes porte sur des principes, des idées, des théories et relève du laboratoire là où l'innovation se rapproche du marché (Mustar \& Penan, 2003).

Dans cette perspective, c'est peut-être de l'invention que devrait relever la didactique des langues dans une période où l'enseignement des langues procède de plus en plus de l'innovation. Cependant en éducation, il n'est pas certain que le changement continu produise des effets prolongés.

\section{BIBLIOGRAPHIE}

Alter, N. (2000). L'innovation ordinaire. Paris : PUF.

Brodin, E. (2002). Interactions entre innovation, technologies de l'information et de la communication et apprentissage institutionnel des langues : l'exemple d'une recherche-action dans des lycées. Thèse de doctorat. Université du Maine. 490 pages.

Chappaz, G. (dir.) (2002). Innovation, éducation et formation. Aix-Marseille : Université de Provence. Cros, F. (dir.) (2001). Politiques de changement et pratiques de changement. Paris : INRP. Ellis, R. (1997). SLA Research and Language Teaching. Oxford : OUP.

Finkelsztein, D. \& Ducros, P. (1996). "Conditions d'implantation et de diffusion d'une innovation scolaire". In Bonami, M. \& Garant, M. (dir.). Systèmes scolaires et pilotage de l'innovation : émergence et implantation du changement. Bruxelles : De Boeck. pp. 32-56.

Flichy, P. de (1994). "Multi-média, objet-valise ou objet-frontière". Futuribles, octobre, nº 191. pp. 3-9.

Fullan, M. G. (1991). The New meaning of educational change. Londres : Cassell.

Gather-Thurler, M. \& Perrenoud, P. (2003). In Groux, D. (dir.). Dictionnaire d'éducation comparée. Paris : L'Harmattan. pp. 315-321.

Génelot, D. (1992). Gérer dans la complexité. Paris : Insep éditions.

Heyworth, F. (1999). Innovative Approaches to the Organisation and Set-up of Language Education. Graz : European Centre for Modern Languages.

Kuhn T. S. (1972). La structure des révolutions scientifiques. Paris : Flammarion. 
Meirieu, P. (2002). "Innover dans l'École : pourquoi ? Comment ?". Conférence au Salon de l'éducation 2002. In Association R.E.V.E.I.L. (Rénover l'École en Valorisant et en Encourageant les Initiatives Locales). Consulté en octobre 2004. http://assoreveil.org/meirieu_3.html

Mustar, P. \& Penan, H. (2003). Encyclopédie de l'innovation. Paris : Économica.

Perrenoud, P. (1998). Réformes scolaires et rénovations de la formation des enseignants : une introuvable synchronisation. Genève : Université de Genève.

Rabardel, P. (1995). Les hommes et les technologies. Paris : Armand Colin.

\section{Sites}

Site Primlangues (nd). Consulté en octobre 2004. http://www.primlangues.education.fr/php/ Site INRP (2004). Banque de données Nova. Consulté en octobre 2004. http://www.inrp.fr/bdd/

Site L'AEF (nd). L'agence éducation emploi formation. Consulté en octobre 2004. http://www.laef.com/

\section{NOTES}

1. On définira le LMD comme une politique ministérielle qui relève d'objectifs internationaux (construction de l'espace européen de l'enseignement supérieur). La démarche a pour but de présenter une offre française de formation plus lisible. Ensuite tout repose sur l'initiative et les propositions des établissements dans le cadre d'un dispositif régulé nationalement par l'évaluation.

2. Les campus numériques sont, actuellement, des dispositifs numériques de formations thématiques regroupant des consortiums d'établissements d'enseignement supérieur.

3. Il s'agit d'une recherche-action impliquant les enseignants de lycée d'une académie (Brodin, 2002).

4. $C=$ changement, $a=$ niveau d'insatisfaction, $b=$ résultat espéré consciemment, $d=$ premiers pas accomplis vers le but poursuivi, $\mathrm{x}=$ coûts divers du changement.

\section{RÉSUMÉS}

L'innovation est un concept souvent utilisé dans le champ de l'éducation, dont les définitions sont à la fois nombreuses et floues. Plutôt que choisir parmi les définitions existantes ou d'en proposer une nouvelle, l'article tente de dégager quelques caractéristiques de l'innovation en formation puis il questionne les spécificités de l'innovation en langues.

Innovation is a frequently used concept in the field of education, with definitions which are both multiple and imprecise. Rather than choosing among existing definitions or suggesting yet another one, the article focuses on a few characteristics of innovation in learning situations. It questions the specificities of innovation in the field of foreign language learning. 
INDEX

Mots-clés : innovation, apprentissage des langues

Keywords : innovation, foreign language learning

\section{AUTEUR}

\section{ÉLISABETH BRODIN}

Élisabeth Brodin enseigne à l'université de Paris 3 et est chargée de mission à la Direction de l'Enseignement Supérieur. Ses domaines de recherche sont l'apprentissage instrumenté des langues, l'innovation et la didactique des langues.

Université Sorbonne Nouvelle Paris 3, 13 rue de Santeuil, 75231 Paris Cedex 05

elisabeth.brodin@wanadoo.fr 